\title{
Fc gamma receptor IIb modulates the molecular Grb2 interaction network in activated B cells
}

\author{
Konstantin Neumann ${ }^{\mathrm{a}, 1}$, Thomas Oellerich ${ }^{\mathrm{a}, 2}$, Ines Heine ${ }^{\mathrm{a}}$, Henning Urlaub ${ }^{\mathrm{b}}$, Michael Engelke ${ }^{\mathrm{a}, *}$ \\ a Georg August University of Göttingen, Institute of Cellular and Molecular Immunology, Humboldtallee 34, 37073 Göttingen, Germany \\ ${ }^{\mathrm{b}}$ Max Planck Institute of Biophysical Chemistry, Bioanalytical Mass Spectrometry, Am Fassberg 11, 37077 Göttingen, Germany
}

\section{A R T I C L E I N F O}

Article history:

Received 15 December 2010

Accepted 14 January 2011

Available online 22 January 2011

\section{Keywords:}

B cell activation

Fc gamma RIIb

$\mathrm{Ca}^{2+}$ mobilization

Grb2

Dok-3

SHIP

SILAC

\begin{abstract}
A B S T R A C T
$B$ cells require signals transduced by the B cell antigen receptor (BCR) to provide humoral adaptive immunity. These signals are modulated by co-receptors like the Fc $\gamma$ receptor IIb (Fc $\gamma$ RIIb) that prevents activation of B cells after co-ligation with the BCR. Positive and negative effectors need to be precisely organized into signaling complexes, which requires adapter proteins like the growth factor receptor-bound protein 2 (Grb2). Here, we address the question how Grb2-mediated signal integration is affected by Fc $\gamma$ RIIb. Our data reveal that concomitant engagement of BCR and Fc $\gamma$ RIIb leads to markedly increased Grb2-mediated formation of ternary protein complexes comprising downstream of kinase-3 (Dok-3), Grb2, and the SH2 domaincontaining inositol phosphatase (SHIP). Consistently, we found Grb2 to be required for full Fc $\gamma$ RIIb-mediated negative regulation. To investigate how FcyRIIb influences the entire Grb2 interactions, we utilized quantitative mass spectrometry to make a differential interactome analysis. This approach revealed a shift of Grb2 interactions towards negative regulators like Dok-3, SHIP and SHP-2 and reduced binding to other proteins like CD19. Hence, we provide evidence that Grb2-mediated signal integration is a dynamic process that is important for the crosstalk between the BCR and its co-receptor Fc $\gamma$ RIIb.
\end{abstract}

(c) 2011 Elsevier Inc. All rights reserved.

\section{Introduction}

Development, survival and activation of $\mathrm{B}$ cells are tightly controlled by the $B$ cell antigen receptor (BCR). The signals mediated by the $\mathrm{BCR}$ need to be precisely regulated to keep the balance between adaptive humoral immunity and tolerance. Hence, positive and negative regulatory effector proteins need to be accurately integrated into the signaling cascade. The BCR comprises an antigen-binding immunoglobulin subunit and an associated Ig- $\alpha / \mathrm{Ig}-\beta$ heterodimer that transduces signals via immuno receptor tyrosine-based activation motifs (ITAMs). Engagement of the BCR leads to phosphorylation of ITAMs and subsequent activation of the spleen tyrosine kinase (Syk) [1]. A bona fide Syk substrate is the adapter protein Src homology 2 (SH2) domain-containing lymphocyte protein of $65 \mathrm{kDa}$ (SLP-65) [2] that upon phosphorylation provides docking sites for the SH2 domains of Bruton's tyrosine kinase (Btk) and phospholipase C- $\gamma 2$ (PLC- $\gamma 2$ )

Abbreviations: FcyRIIb, fragment crystalline of gamma chain receptor IIb; Grb2, growth factor receptor-bound protein 2; Dok-3, downstream of kinase-3; SHIP, SH2 domain-containing inositol phosphatase; SILAC, stable isotope labeling of amino acids in cell culture.

* Corresponding author. Tel.: +49 551 3913858; fax: +49 551395843.

E-mail address: mengelk@gwdg.de (M. Engelke).

1 Current address: Technical University of Munich, Institute of Molecular Immunology, Klinikum Rechts der Isar, Ismaninger Str. 22, 81675 Munich, Germany.

2 Current address: Georg August University of Göttingen, Department of Nephrology and Rheumatology, Robert-Koch-Strasse 40, 37075 Göttingen, Germany.
$[3,4]$. Formation of this trimolecular complex leads to mobilization of $\mathrm{Ca}^{2+}$ ions and activation of the $\mathrm{NF}_{\kappa}-\mathrm{B}$ and MAPK pathways [5].

$\mathrm{BCR}$ engagement can trigger opposed cellular fates like activation and differentiation or anergy and apoptosis, which depends on the developmental status of the B cell and concomitant engagement of other receptors [6]. Some of these receptors deliver co-activatory stimuli. CD19, for example, lowers the activation threshold of B cells by recruiting phosphoinositol-3-kinase (PI3K) $[7,8]$. PI3K catalyzes the production of phosphatidyl-3,4,5-trisphosphate (PIP3) at the cytosolic leaflet of the plasma membrane. PIP3 is a docking site for the Pleckstrin homology (PH) domains of Btk and PLC- $\gamma 2$ and hence stabilizes their plasma membrane localization [9,10]. Limitation of BCR-induced activation is mediated by receptor proteins that harbor immuno receptor tyrosine-based inhibition motifs (ITIMs). ITIMs recruit negative regulatory effector proteins via their SH2 domains [11]. For example the sialic acid-binding immunoglobulin [Ig]-like lectin-2 (Siglec-2 or $\mathrm{CD} 22$ ) recruits the $\mathrm{SH} 2$ domain-containing protein tyrosine phosphatase-1 (SHP-1) which dephosphorylates several targets like Ig- $\alpha$, Ig- $\beta$ and SLP-65 [12].

Another ITIM-containing receptor that has been extensively studied in the past is Fc $\gamma$ receptor IIb (Fc $\gamma$ RIIb), which gets co-engaged if the BCR recognizes antigen that is already bound by $\operatorname{IgG}$ [13]. The phosphorylated Fc $\gamma$ RIIb ITIM recruits the SH2 domain-containing inositol 5-phosphatase (SHIP) which hydrolyzes PIP3 and hence destabilizes the plasma membrane localization of $\mathrm{Ca}^{2+}$-mobilizing enzymes [14,15]. This process is believed to prevent the production of new antibodies to a given antigen 
in a situation where class-switched and affinity-optimized antibodies are already available. Mice deficient for Fc $\gamma$ RIIb mount augmented humoral immune responses to both thymus-dependent and thymus-independent antigens [16]. Moreover, absence of Fc $\gamma$ RIIb renders mice susceptible for autoimmunity [17]. Interestingly, CD19, CD22 and Fc $\gamma$ RIIb bind to the adapter protein growth factor receptor-bound protein 2 (Grb2) [18-20]. It has been shown that Fc $\gamma$ RIIb-mediated SHIP recruitment is strongly compromised in Grb2-deficient DT40 cells [19]. However, the exact function of Grb2 in this context is not entirely understood.

Several studies elucidated the importance of Grb2 to regulate BCRmediated signal transduction also independently of co-receptors. In complex with the adapter protein downstream of kinase-3 (Dok-3) Grb2 attenuates PLC- $\gamma 2$-dependent production of inositol-1,4,5-trisphosphate [21]. Dok-3-deficient mice have increased serum IgM titers and enhanced humoral immune responses to $\mathrm{T}$ cell-independent antigens [22]. The regulatory function of Grb2 differs among developmental B cell stages. While in BCR-stimulated immature B cells Dok-3 appears to be the main Grb2-binding protein in mature B cells Grb2 is differently recruited by the transmembrane adapter protein linker for activation of T cells 2 (LAT2), which abolishes Grb2-mediated inhibition [23]. In memory B cells that express IgG- or IgE-containing BCRs Grb2 binds to the immunoglobulin tail tyrosine (ITT) motif which provides a co-stimulatory signal that appears to be essential for survival of these cells [24].

Recently, the Grb2 interactome in activated Bal17 B cells was elucidated by quantitative mass spectrometry analysis [25]. This study revealed that Grb2 interacts with a plethora of inhibitory and activatory signaling proteins. Notably a number of modulators of the PI3K signaling pathway were found among the 27 identified Grb2-associated proteins. These include positive regulators like the B cell adapter for PI3K (BCAP), Vav (seventh letter in the Hebrew alphabet) and the PI3K subunit $\mathrm{p} 85$ as well as negative regulators like SHIP-1/-2 and SHP-1/-2 [25]. These data imply a pivotal role of $\mathrm{Grb} 2$ in integrating signaling regulators. It is currently not understood whether co-receptors like Fc $\gamma$ RIIb utilize the molecular Grb2 interaction network to inhibit BCR-mediated signaling.

In this study we elucidated the impact of Fc $\gamma$ RIIb on Grb2-mediated signal integration in activated B cells by combining biochemical and quantitative mass spectrometry analysis. Our approach revealed that co-engagement of BCR and Fc $\gamma$ RIIb augments the formation of ternary Dok-3/Grb2/SHIP protein complexes. Moreover, we could show by differential interactome analysis that the molecular Grb2 interaction network drastically differs in BCR- versus BCR/Fc $\gamma$ RIIb-activated cells. Our data imply that Grb2 integrates more positive regulators of PI3K in BCR-stimulated cells whereas after co-engagement of Fc $\gamma$ RIIb the negative regulators SHIP-1/-2 and SHP-2 are prominently bound to Grb2.

\section{Material and methods}

\subsection{Cells, abs and reagents}

Bal17.TR (kindly provided by Dr. Tony de Franco, University of California, San Francisco) were cultured in RPMI 1640 containing 10\% FCS, $2 \mathrm{mM}$ L-glutamine, $2 \mathrm{mM}$ pyruvate, $50 \mu \mathrm{M}$ ß-ME, and antibiotics (cell culture reagents were purchased from Invitrogen). For SILAC experiments we used RPMI lacking arginine and lysine (Thermo Scientific). For retroviral infection of Bal17.TR cells we used a pMSCV-puro vector (Clontech) and constructs encoding mouse Grb2 [23] or the OneSTrEPtagged variant [25]. BCR stimulation was performed for indicated times using $10 \mu \mathrm{g} / \mathrm{ml} \mathrm{F}(\mathrm{ab})_{2}$ fragments of goat anti-mouse IgM or $15 \mu \mathrm{g} / \mathrm{ml}$ complete goat anti-mouse IgM (Jackson Immuno Research Laboratories). Cells were lysed as described in [21]. For immunoprecipitation rabbit antiDok-3 S-20 (Santa Cruz Biotechnology) was used. For Western blot analysis we used anti-pTyr 4G10, anti-Grb2 3F2 (Millipore), anti-SHIP D1163, anti-Src $\mathrm{Y}^{416}$, Lyn $\mathrm{Y}^{507}$ (Cell Signaling Technology), and anti-Lyn 44 (Santa Cruz Biotechnology). For detection of mouse Dok-3 a polyclonal rabbit antiserum was kindly provided by Dr. Andre Veillette (Institut de recherches cliniques de Montréal). Recombinant Grb2-GST was prepared as described previously [26].

\subsection{Mass spectrometric analysis}

For stable isotope labeling of amino acids in cell culture (SILAC) Bal17. TR cells expressing a OneSTrEP-tagged Grb2 were cultured in RPMI 1640 medium devoid of arginine and lysine (Pierce) supplemented with $10 \%$ dialyzed FCS (Invitrogen), 1 mM pyruvate, and 4 mM glutamine. "Heavy" medium was supplemented with ${ }^{2} \mathrm{D}_{4},{ }^{12} \mathrm{C}_{6},{ }^{14} \mathrm{~N}_{2}$ lysine and ${ }^{13} \mathrm{C}_{6},{ }^{14} \mathrm{~N}_{4}$ arginine (Cambridge Isotopes) and "light" medium was prepared by adding equimolar levels of the corresponding non-labeled amino acids (Sigma-Aldrich). For affinity purification of OneSTrEP-tagged Grb2 $10^{8}$ cells from the respective cultures were stimulated with either goat antimouse $\mathrm{F}(\mathrm{ab})_{2}$ fragments to engage the $\mathrm{BCR}$ alone or with complete rabbit anti-mouse to co-engage BCR and Fc $\gamma$ RIIb. Cleared cellular lysates were incubated with $200 \mu \mathrm{l}$ of Strep-Tactin Superflow matrix (Iba BioTAGnology) for $1 \mathrm{~h}$ at $4{ }^{\circ} \mathrm{C}$. After washing the immobilized Grb2 was eluted by incubating with $500 \mu \mathrm{l}$ of D-desthiobiotin elution buffer (IBA BioTAGnology), pooled at a 1:1 ratio, and concentrated with ultrafiltration spin columns (Sartorius). Proteins were separated by one-dimensional SDSPAGE (4-12\% NuPAGE Bis-Tris Gel, Invitrogen) and each lane of the Coomassie-blue-stained gel was cut into 23 slices. All slices were reduced with $10 \mathrm{mM}$ DTT for $55 \mathrm{~min}$ at $56{ }^{\circ} \mathrm{C}$, alkylated with $55 \mathrm{mM}$ IAA for 20 min at $26^{\circ} \mathrm{C}$ and digested with modified trypsin (Promega) overnight at $37^{\circ} \mathrm{C}$. Following digestion peptides were injected into a $\mathrm{C} 18$ precolumn $(1.5 \mathrm{~cm}, 360 \mu \mathrm{m}$ o.d., $150 \mu \mathrm{m}$ i.d., Reprosil-Pur $120 \AA ̊, 5 \mu \mathrm{m}$, C18-AQ Dr. Maisch $\mathrm{GmbH}$ ) at a flow rate of $10 \mu \mathrm{l} / \mathrm{min}$. Bound peptides were eluted and then separated on a C18 capillary column $(15 \mathrm{~cm}$, $360 \mu \mathrm{m}$ o.d., $75 \mu \mathrm{m}$ i.d., Reprosil-Pur $120 \AA$ A, $5 \mu \mathrm{m}, \mathrm{C} 18-\mathrm{AQ}$ Dr. Maisch $\mathrm{GmbH}$ ) at a flow rate of $300 \mathrm{nl} / \mathrm{min}$, with a gradient from $7.5 \%$ to $37.5 \%$ ACN in $0.1 \%$ formic acid for 60 min using an Agilent 1100 nano-flow LC system (Agilent Technologies) coupled to a LTQ-Orbitrap XL hybrid mass spectrometer (Thermo Electron). MS conditions were set as following: spray voltage, $1.8 \mathrm{kV}$; heated capillary temperature, $150^{\circ} \mathrm{C}$; normalized collision-induced dissociation (CID) collision energy 37.5\% for MS/MS in LTQ. An activation $q=0.25$ and activation time of $30 \mathrm{~ms}$ were used. The mass spectrometer was running in the data dependent mode. It automatically switched between MS and MS/MS acquisition. Survey MS spectra were acquired in the Orbitrap (m/z 350-1600) with the resolution set to 30,000 at $\mathrm{m} / \mathrm{z} 400$ and automatic gain control target at $5 \times 10^{5}$. The five most intense ions were sequentially isolated for CID MS/MS fragmentation and detection in the linear ion trap. Ions with single and unrecognized charge states were not included.

For quantification raw data were analyzed using the MaxQuant software (Version 1.0.12.31) in combination with Mascot search engine for peptide and protein identifications (Version 2.2.04, Matrix Science). IPI Mouse (Version 3.47) was used as a mus musculus sequence database. MS/ MS peak lists were filtered to contain at most six peaks per $100 \mathrm{Da}$ intervals and searched against Mascot server. The MS mass tolerance was set to $7 \mathrm{ppm}$ and MS/MS mass tolerance was set to $0.6 \mathrm{Da}$. Up to three missed cleavages of trypsin were allowed. Oxidized methionine and cysteine carbamidomethylation were searched as variable modifications. The modifications corresponding to arginine and lysine labeled with heavy stable isotopes could be treated as fixed modifications in the Mascot search, if applicable, after identification of SILAC pairs by MaxQuant. The false positive rate was set to $1 \%$ at the peptide level, the false discovery rate was set to $1 \%$ at the protein level and the minimum required peptide length was set to six amino acids.

\subsection{Calcium measurements}

$10^{6}$ cells were loaded in $700 \mu \mathrm{l}$ RPMI containing $5 \%$ FCS, $1 \mu \mathrm{M}$ Indo1-AM (Molecular Probes) and 0.015\% Pluronic F127 (Molecular Probes) at $30{ }^{\circ} \mathrm{C}$ for $25 \mathrm{~min}$. Subsequently, the cell suspension was diluted two-fold with RPMI $10 \%$ FCS and incubated for 10 min at $37^{\circ} \mathrm{C}$. 
Cells were washed and prepared for measurements as described earlier [21]. Changes in the ratio of fluorescence intensities at $405 \mathrm{~nm}$ and $510 \mathrm{~nm}$ were monitored on a LSRII flow cytometer (Becton Dickinson) and analyzed with Flowjo (TriStar).

\section{Results}

3.1. Co-engagement of the BCR and FcyRIIb leads to increased formation of a ternary Dok-3/Grb2/SHIP complex

To examine the interdependence of Fc $\gamma$ RIIb and Grb2 interactions we first analyzed the Dok-3/Grb2 association. Previous findings of our laboratory emphasize Dok-3 to be the main BCR-proximal Grb2interacting protein under positive signaling conditions [21]. Moreover, Dok-3 and Grb2 bind to the Fc $\gamma$ RIIb effector SHIP [27-29] implying that Dok-3/Grb2 might play a role under negative signaling conditions, too. To compare BCR versus BCR/Fc $\gamma$ RIIb engagement we stimulated Bal17 B cells with two different protocols. To engage the BCR in the absence of Fc $\gamma$ RIIb signals we used anti-IgM F(ab)2 that lacks the Fc $\gamma$ RIIb-binding part. This procedure revealed very similar results like blocking the IgG binding site in Fc $\gamma$ RIIb with anti-Fc $\gamma$ R antibody (data not shown). For concomitant stimulation of BCR and FcyRIIb we treated cells with complete anti-IgM antibodies that bind to the Fc $\gamma R$ IIb via its Fc part. We then prepared cellular lysates from these cells, immunopurified Dok-3, and subjected the samples to Western Blot analysis. Probing the membrane with anti-Grb2 antibodies revealed an increased amount of co-purified Grb2 after BCR/Fc $\gamma$ RIIb co-stimulation (Fig. 1A, first panel, lanes 5-7) compared to BCR engagement alone (lanes 2-4). This finding correlates with Dok-3 tyrosine phosphorylation (Fig. 1A, second panel). We looked for co-purified SHIP in our setup and anti-SHIP immunoblotting indeed revealed a stronger Dok-3/SHIP association in BCR/ FcyRIIb co-stimulated cells (Fig. 1A, third panel). Similar amounts of Dok-3 were confirmed as depicted in the fourth panel of Fig. 1A. To exclude that the increase in Dok-3 phosphorylation is merely due to augmented Lyn kinase activity, we assessed Lyn activation by Western blot analysis. We used phospho-specific antibodies that recognize the activating (Y397) and inhibitory (Y507) tyrosines, which are conserved in all Src kinases. This approach revealed no significant differences in Lyn activation in response to BCR crosslinking versus BCR/Fc $\gamma$ RIIb co- crosslinking (Fig. 1B, first and second panels). Equal loading was confirmed by anti-actin immunoblotting (Fig. 1B, third panel). These results strongly imply that Fc $\gamma$ RIIb utilizes Dok-3 by enhancing its tyrosine phosphorylation, which is not caused by a generally increased Lyn activity.

Our data show that the association of Dok-3 with both Grb2 and SHIP is augmented by co-crosslinking of BCR and Fc $\gamma R$ RIIb. Given the reported direct binding of Grb2 to SHIP this suggests formation of a ternary complex comprising Dok-3, SHIP, and Grb2. To test this, we made use of a Grb2-deficient B cell line (Bal17.TR) [30] that was either transfected with a Grb2 expression vector or empty vector as control. We performed again anti-Dok-3 immunopurifications and found that the amount of copurified SHIP was strongly reduced in the absence of Grb2 expression (Fig. 2A, upper panel). This Grb2 dependency was most obvious when Dok-3 was immunopurified after co-engagement of BCR and Fc $\gamma$ RIIb (lanes 5 and 6) or treatment with the phosphatase inhibitor pervanadate (lanes 7 and 8). The differences are neither due to varying amounts of purified Dok-3 (Fig. 2A, middle panel) nor to unequal SHIP expression (Fig. 2A, lower panel). Note that pervanadate treatment leads to additional posttranslational modifications of Dok-3, which is evident in slower migrating bands in the anti-Dok-3 immunoblot. To further confirm the importance of Grb2 for the Dok-3/SHIP association, we reconstituted Grb2 in vitro. Bal17 cells were treated with pervanadate to induce maximal tyrosine phosphorylation of SHIP and Dok-3. To cellular lysates recombinant GST-Grb2 or GST only as control was added. After immunoprecipitation of Dok-3 we detected co-purified SHIP by Western blot analysis. As shown in Fig. 2B, SHIP was only detected in the presence of GST-Grb2 (lane 3). These data show that Grb2 stabilizes the association of Dok-3 and SHIP leading to the formation of a ternary complex.

To test whether this finding has consequences for downstream signaling processes we monitored $\mathrm{Ca}^{2+}$ mobilization in the Bal17.TR transfectants described above. From Fig. $2 \mathrm{C}$ it is apparent that Grb2 attenuates $\mathrm{Ca}^{2+}$ flux after engagement of BCR alone (left graph). This is in line with our previous findings in DT40 and K46 cells [23]. However, the difference in $\mathrm{Ca}^{2+}$ profiles of Bal17.TR and reconstituted cells is significantly more pronounced after BCR/Fc $\gamma$-RIIb stimulation (Fig. 2C, right graph). While $\mathrm{BCR} / \mathrm{Fc} \gamma \mathrm{RIIb}$ co-engagement compared to $\mathrm{BCR}$ stimulation already in absence of Grb2 expression limits the duration of
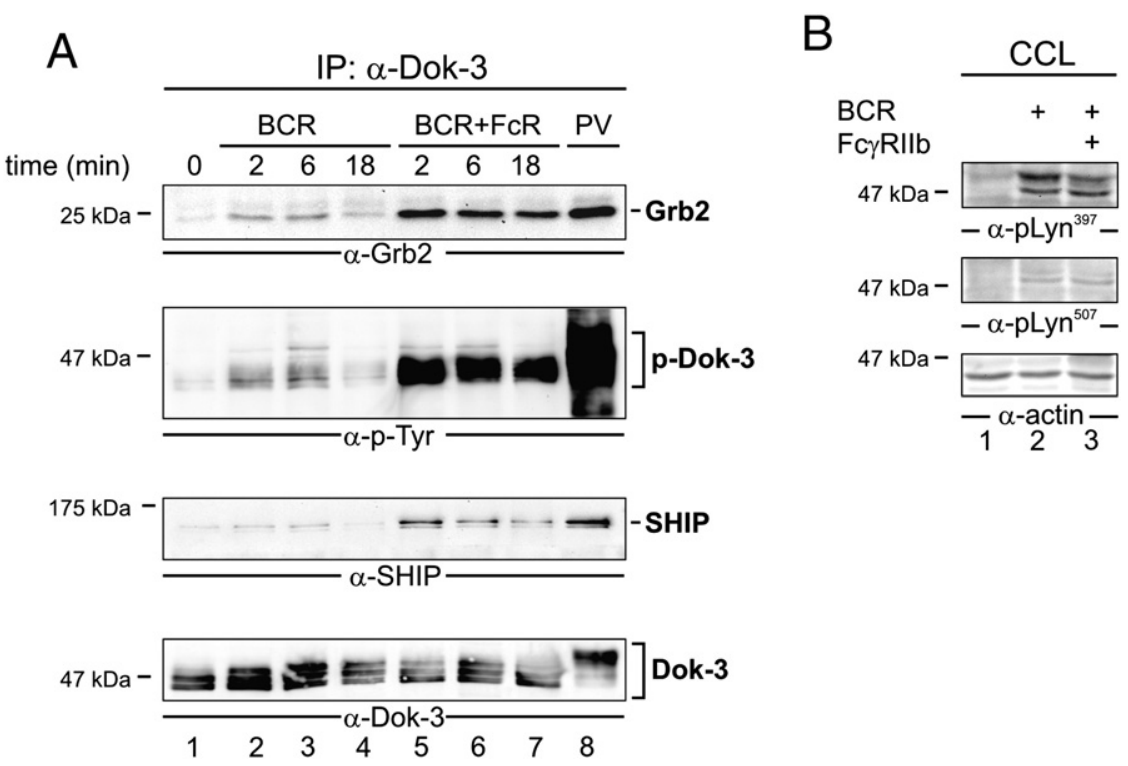

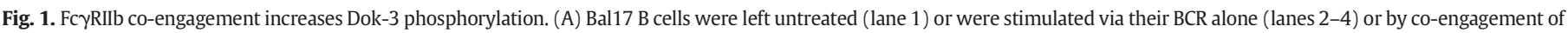

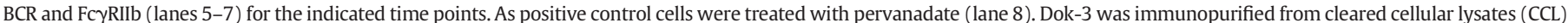

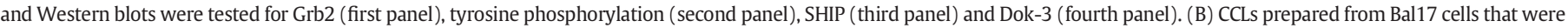

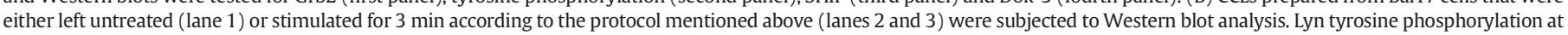

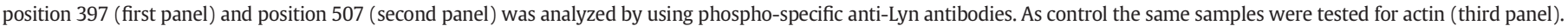


A
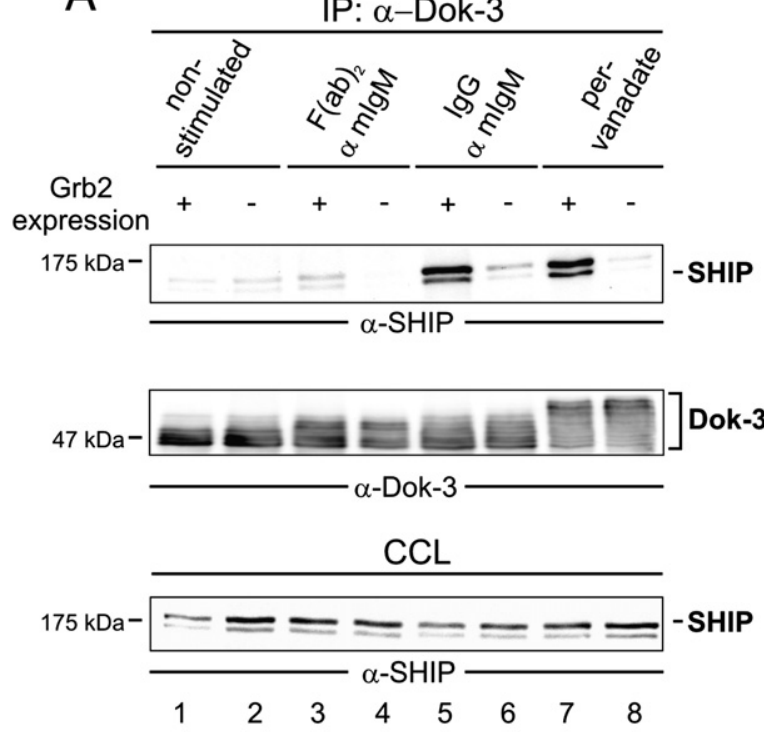

C

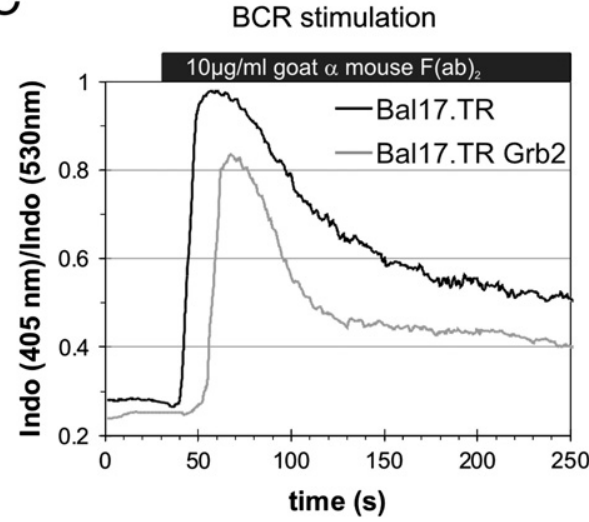

B

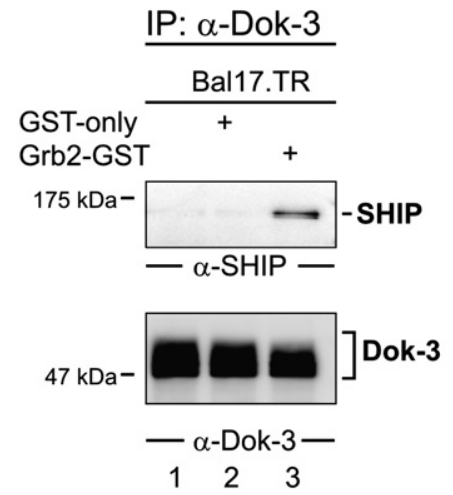

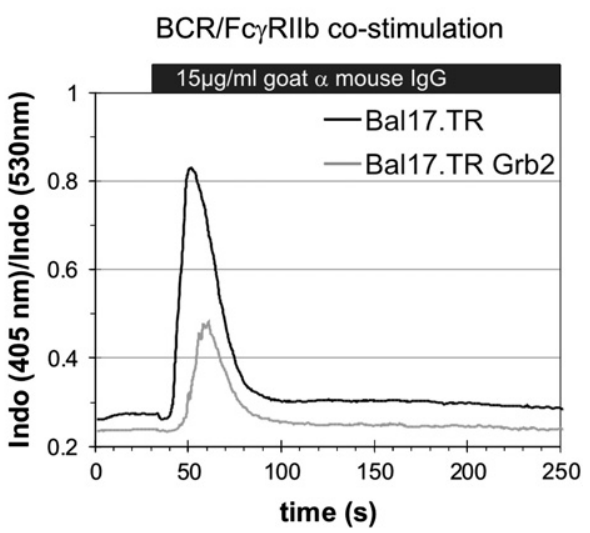

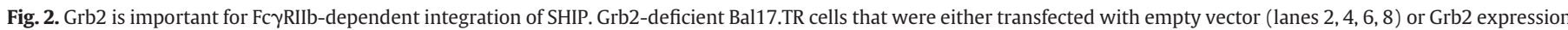

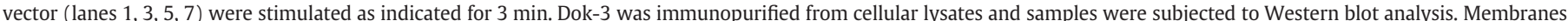

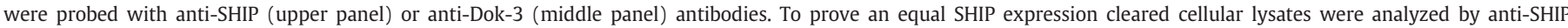

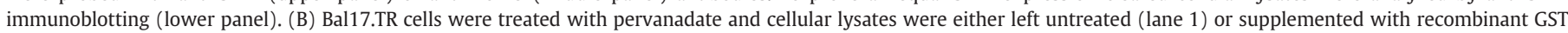

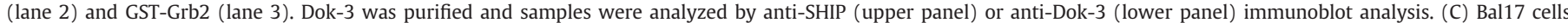

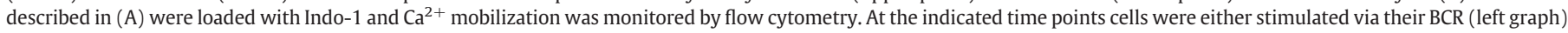
or by BCR/Fc $\gamma$ RIIb co-engagement (right graph).

$\mathrm{Ca}^{2+}$ mobilization (compare black lines) in reconstituted cells concomitant engagement of BCR and FcyRIIb compromises the $\mathrm{Ca}^{2+}$ response drastically (compare gray lines). These results clearly confirm that Grb2 is also important for signal regulation under negative signaling conditions.

\subsection{Fc $\gamma$ RIIb co-ligation shifts the Grb2 interactome to negative regulatory proteins}

Our results described above show that Fc $\gamma$ RIIb co-engagement markedly increases formation of Dok-3/Grb2/SHIP complexes. Given the more than $20 \mathrm{Grb2}$-binding proteins that were recently identified in our laboratory [25] we now analyzed the impact of Fc $\gamma$ RIIb on Grb2 interactions in more detail and employed differential interactome analysis by quantitative mass spectrometry. Briefly, we metabolically labeled Bal17.TR cells expressing OneSTrEP-tagged Grb2 in SILAC medium containing lysine and arginine with either "light" isotopes $\left({ }^{12} \mathrm{C}_{6},{ }^{14} \mathrm{~N}_{2}\right.$-Lys; ${ }^{12} \mathrm{C}_{6},{ }^{14} \mathrm{~N}_{4}$-Arg) or "heavy" isotopes $\left({ }^{2} \mathrm{D}_{4},{ }^{12} \mathrm{C}_{6},{ }^{14} \mathrm{~N}_{2}\right.$-Lys; ${ }^{13} \mathrm{C}_{6},{ }^{14} \mathrm{~N}_{4}$-Arg). The cells were then stimulated via their BCR (light isotopes) or by BCR/Fc $\gamma$ RIIb co-engagement (heavy isotopes) and Grb2 was purified from cellular lysates via a Streptactin ${ }^{\mathrm{TM}}$ matrix. Samples were pooled at a 1:1 ratio and after one-dimensional PAGE and trypsin digestion resulting peptides were identified by liquid chromatography-coupled tandem mass spectrometry (Fig. 3A). The metabolic labeling enables an unbiased relative quantification of copurified proteins from differently treated cells as each identified peptide occurs with two distinct $m / z$ (mass over charge) values. We were able to identify $26 \mathrm{Grb2}$-binding proteins (Table 1 ) that match the published Grb2 interactions [25]. We identified peptide pairs of

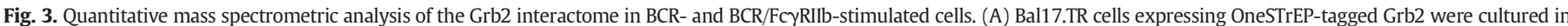

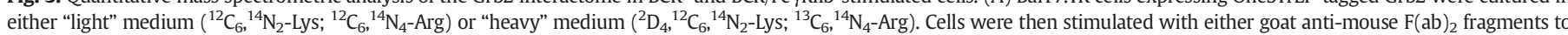

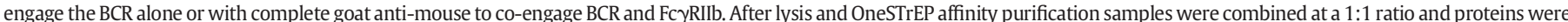

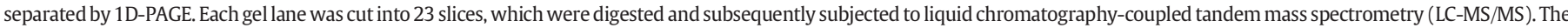

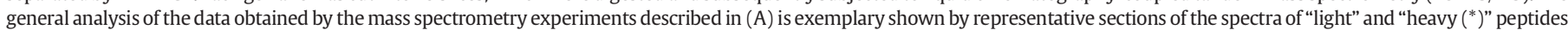
derived from proteins Grb2 (B) and SHP-2 (C). The difference in the $m / z$ values between the "light" and the "heavy" peptides are indicated within the spectra. 
A

A Bal17.TR

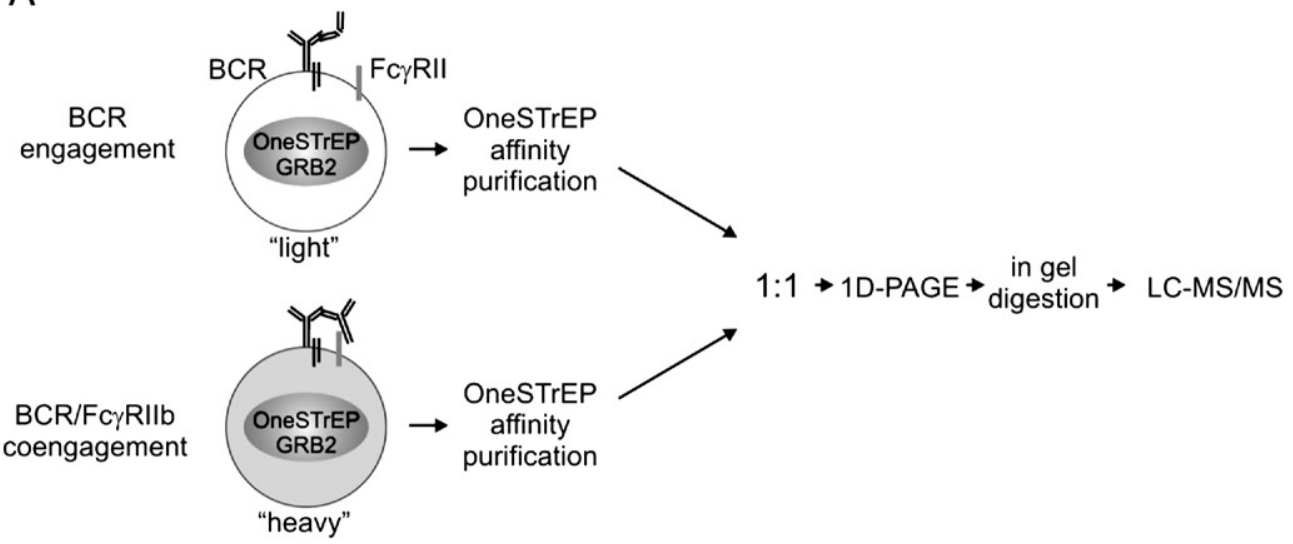

B

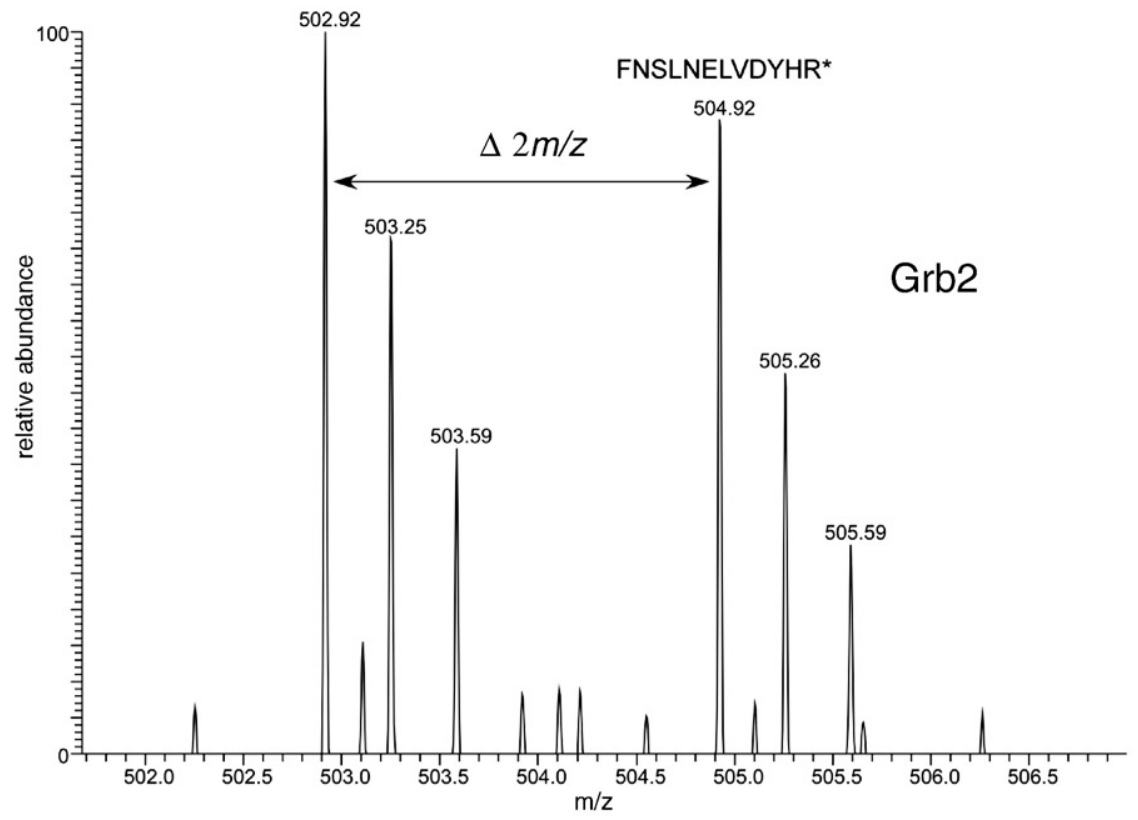

C

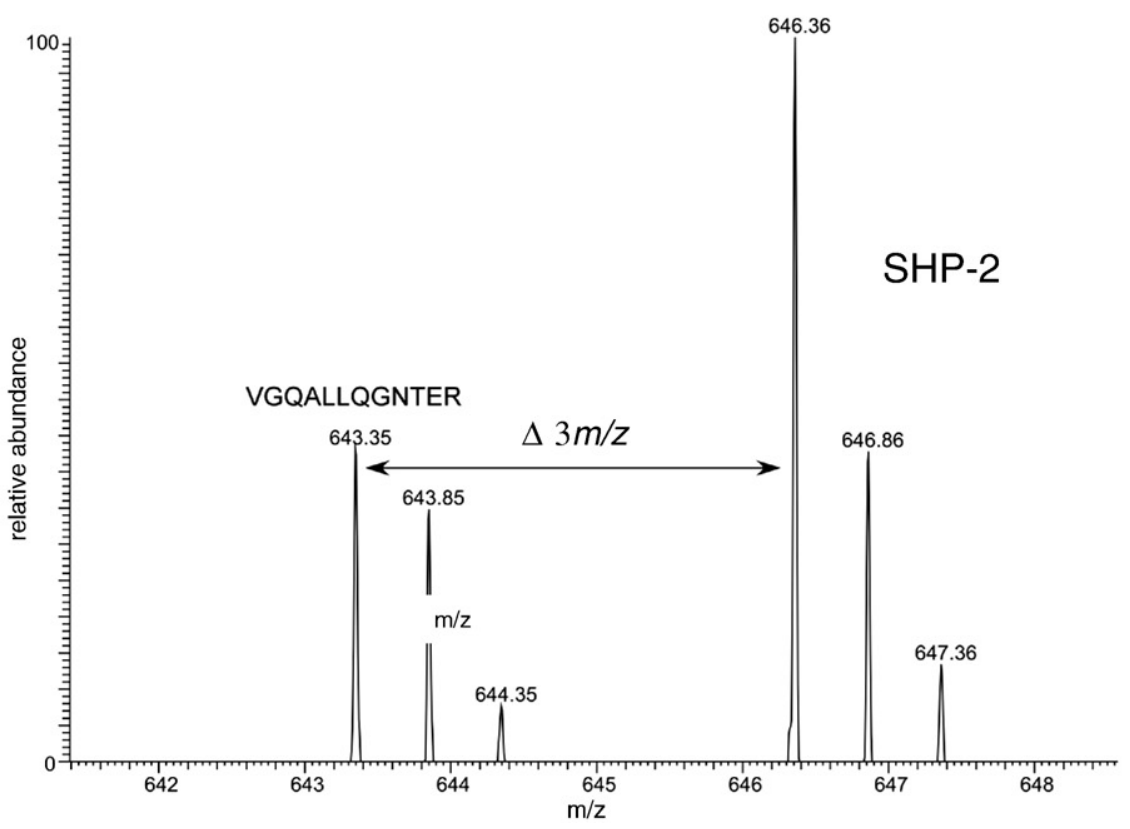


Table 1

The interactome of Grb2 in B cells. The listed proteins were co-purified with Grb2, identified in at least two of three independent experiments, and match the published Grb2-interacting proteins [25]. The accession numbers correspond to the Universal Protein Resource (UniProt) data base. The detailed statistics of the mass spectrometric analysis are available in the supplementary data.

\begin{tabular}{lll}
\hline Identified protein & Accession number & Function \\
\hline SHIP-1 & Q9ES52 & inositol 5'-phosphatase \\
SHIP-2 & Q6P549 & inositol 5'-phosphatase \\
SHP-1 & P29351 & protein tyrosine phosphatase \\
SHP-2 & P35235 & protein tyrosine phosphatase \\
PTP-PEST & P35831 & protein tyrosine phosphatase \\
HPK-1 & P70218 & MAP4K \\
PTPRA & P18052 & receptor-type protein tyrosine phosphatase \\
Dok-3 & Q9QZK7 & cytosolic adapter protein \\
SHC & P98083 & cytosolic adapter protein \\
SLP-65 & Q9QUN3 & cytosolic adapter protein \\
Gab-2 & Q9Z1S8 & cytosolic adapter protein \\
BCAP & Q9EQ32 & cytosolic adapter protein \\
SNX18 & Q91ZR2 & cytosolic adapter protein \\
CD19 & P25918 & transmembrane adapter protein \\
\hline
\end{tabular}

these interactors as exemplarily shown in Fig. 3B and C for a Grb2 and SHP-2 peptide, respectively. We then calculated the relative abundance of each corresponding pair of peptides (for detailed statistics see supplementary data). Heavy/light ratio values of $>1$ reflect increased and values of $<1$ reflect decreased amounts of co-purified proteins in the BCR/Fc $\gamma$ RIIb-co-crosslinked sample compared to the BCR-stimulated sample. In Fig. 4 , the mean ratios of two or three $\left(^{*}\right)$ independent experiments are illustrated as a bar plot. We identified three groups of proteins whose interaction with Grb2 is increased, decreased or not affected by concomitant stimulation of BCR and Fc $\gamma$ RIIb. The mass spectrometric approach confirmed our biochemical analysis described above as Dok-3 belongs to the group of proteins with augmented binding to Grb2 (ratio $1.48 \pm 0.08$ ). The increase of Grb2 association after Fc $\gamma$ RIIb-co-engagement is more pronounced for the negative regulators SHIP- 1 (ratio $1.88 \pm 0.21$ ), SHIP-2 (ratio $2.07 \pm$ 0.14 ), and SHP-2 (ratio $1.68 \pm 0.12$ ), which fits the limiting role of Fc $\gamma$ RIIb in BCR signaling. Consistently, the positive regulators P85 $\alpha / \beta$ (ratio $0.65 \pm 0.11$ ) and Vav-1 (ratio $0.74 \pm 0.12$ ) belong to the group of proteins with Fc $\gamma$ RIIb-dependent reduction within the Grb2 interactome. This also applies to PTP-PEST (ratio $0.76 \pm 0.11$ ) as well as the ubiquitin ligases Cbl-b (ratio 0.76 \pm 0.16 ) and c-Cbl (ratio $0.69 \pm 0.13$ ). Notably Grb2 recruitment by other transmembrane proteins is affected by Fc $\gamma$ RIIb co-engagement, too. We found diminished binding of Grb2 to CD19 (ratio 0.6 \pm 0.10 ), CD72 (ratio $0.85 \pm 0.04$ ), and the receptor-type protein tyrosine phosphatase $\alpha$ (PTPRA) (ratio $0.71 \pm 0.09$ ). Moreover, Grb2 binding to putative regulators of the cytoskeleton dynamics and cellular trafficking like sorting nexin-18 (Snx-18) (ratio 0.64 \pm 0.02 ), ASAP-1 (Arf-GAP containing SH3 domain, ankyrin repeats, and $\mathrm{PH}$ domain) (ratio $0.62 \pm 0.03$ ), and the RhoGAP ARHGAP-12 (ratio $0.76 \pm$ 0.21 ) is decreased. The group of proteins that are purified from BCR- and BCR/Fc $\gamma R$ IIIb-stimulated cells in similar amounts include SLP-65, BCAP, HPK-1 (hematopoietic progenitor kinase-1), Shc, SHP-1, Grb2-associated binder 2 (Gab-2), Dynamin-2/3, and Sos-1/2 (son of sevenless-1 and 2). Hence, our quantitative mass spectrometric approach shows for the first time a shift in the global interactions of a protein in response to cocrosslinking of an inhibitory co-receptor further indicating the importance of Grb2 for signal integration in B cells.

\section{Discussion}

The fundamental importance of Fc $\gamma$ RIIb in keeping the balance between humoral immunity and autoimmune responses has mainly been attributed to the recruitment of SHIP. Our approach confirms the importance of SHIP and provides evidence for further alterations in the signaling cascade. We show that major parts of the molecular Grb2 interaction network are modified by FcyRIIb co-engagement which

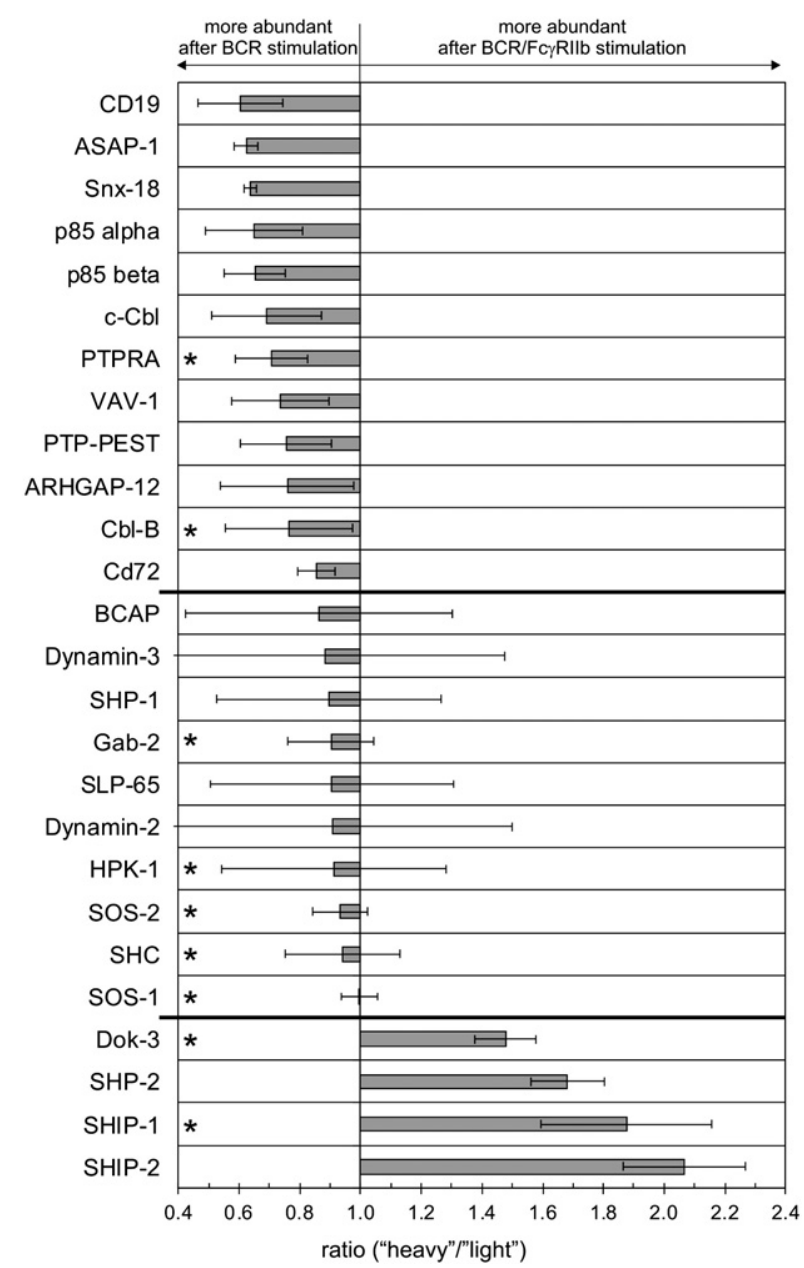

Fig. 4. Fc $\gamma$ RIIb co-engagement changes the Grb2 interaction network. The quantitative mass spectrometry data of two or three $\left({ }^{*}\right)$ independent experiments were calculated as depicted in Fig. 3. The heavy/light ratios are normalized to Grb2 (ratio $=1$ ) and error bars reflect the mean deviation. Values of $>1$ reflect increased and values of $<1$ reflect decreased amounts of co-purified proteins in the BCR/Fc $\gamma$ RIIb-co-stimulated sample compared to the BCR-stimulated sample. The bold lines indicate the threshold for ratios that are significantly greater or smaller than 1 .

results in the differential integration of various regulators into the BCR signaling cascade. Consistent with these data the inhibitory function of FcyRIIb appears to require Grb2 as obvious from our $\mathrm{Ca}^{2+}$ flux analysis.

The Grb2-mediated signal integration starts with the SH2 domaindependent relocalization of Grb2 to the plasma membrane. Despite a number of known interactors for the Grb2 SH2 domain, the mandatory Grb2-recruiting protein in BCR-stimulated cells appears to be Dok-3 because BCR-induced Grb2 relocalization hardly occurs in Dok-3-deficient DT40 B cells [21]. Our data show that BCR/Fc $\gamma$ RIIb co-stimulation evokes a more pronounced tyrosine phosphorylation of Dok-3 than BCR stimulation alone. This is not related to an altered Lyn activity, which is consistent with earlier reports [31]. More likely Fc $\gamma$ RIIb appears to control the Dok-3 microlocalization at the plasma membrane that increases the efficiency of its phosphorylation. A similar observation has been reported for Dok-1 that is also stronger phosphorylated after BCR/Fc $\gamma$ RIIb stimulation leading to increased recruitment of the Ras GTPase-activating protein (RasGAP) and subsequent inhibition of the MAPK pathway [32]. From these data it appears that the most BCR-proximal PTK Lyn is utilized to mediate negative regulatory functions of Fc $\gamma$ RIIb leading to augmented formation of Dok-1/RasGAP and Dok-3/Grb2 complexes. In the context of Grb2 this causes increased recruitment to the plasma membrane, which has fundamental consequences for further Grb2-mediated signal integration.

It has been reported earlier that the mouse ortholog of Fc $\gamma$ RIIb itself harbors a binding site for the Grb2 SH2 domain and that SHIP-1 
recruitment in absence of Grb2 is impaired [19]. However, we could not specifically identify Fc $\gamma$ RIIb within the Grb2 interactome as Fc $\gamma$ RIIb peptides were found only in one out of three experiments (see supplementary data). One could speculate that this is due to incomplete cell lysis but other transmembrane proteins like CD72 and CD19 were identified under the given conditions. More likely, the interaction with Fc $\gamma$ RIIb might not be very prominent within the Grb2 interactome. Given the very strict parameter settings for the analysis of the mass spectrometry data those weakly co-purified proteins might be lost. In this context it is noteworthy that the consensus Grb2 binding site of mouse Fc $\gamma$ RIIb is not conserved among species leaving the role of this motif for SHIP-1 recruitment unclear. Our results imply that Grb2 stabilizes SHIP-1/Fc $\gamma$ RIIb complexes indirectly in alliance with Dok-3. We and others showed earlier that the Dok-3 PTB domain binds to SHIP-1 $[21,28,33]$. Consistent with these reports, which emphasize the importance of the Grb2 binding site in Dok-3 for SHIP1 binding, we found Grb2 to be essential for this interaction. A similar ternary protein complex has been described for Shc, Grb2, and SHIP-1 [30]. However, Fc $\gamma$ RIIb co-engagement with the BCR appears to affect mainly Dok-3/Grb2/SHIP-1 complexes as we found Grb2 association with Shc to be unaltered. Therefore we propose that Fc $\gamma$ RIIbdependent recruitment of SHIP-1 is a bidentated process. First, binding of the SHIP-1 SH2 domain to the phosphorylated Fc $\gamma$ RIIb ITIM and second, association with the plasma membrane resident Dok-3. Dok-3 and SHIP-1 are connected by Grb2 via its SH2 and Cterminal SH3 domain, respectively [19], leading to stable plasma membrane localization of SHIP. Consistent with this model we copurified markedly increased amounts of SHIP-1 with Dok-3 from BCR/ Fc $\gamma$ RIIb-co-stimulated cells. Our differential interactome analysis revealed a similar characteristic for the homologue SHIP-2. Indeed it has been proposed earlier, that SHIP-2 is also recruited to the Fc $\gamma$ RIIb ITIM [34]. However, the role of SHIP-2 in BCR or FcyRIIb signaling remains elusive to date.

Our quantitative mass spectrometric analysis unveiled that Fc $\gamma$ RIIb co-engagement has considerable consequences for Grb2-mediated signal integration beyond SHIP recruitment. Prominently upregulated in BCR/ Fc $\gamma$ RIIb-co-stimulated cells is the $\mathrm{SH} 2$ domain-containing tyrosine phosphatase-2 (SHP-2). Unlike for its relative SHP-1 the exact role of SHP-2 in BCR signaling is unclear. Beside a proline rich motif interacting with the C-terminal Grb2 SH3 domain SHP-2 harbors a consensus binding motif for the Grb2 SH2 domain that mediates adapter function leading to Ras activation downstream of a number of cytokine and growth factor receptors but neither the BCR nor FcyRIIb [35-37].
Indications for a function of SHP-2 downstream of antigen receptors derive from studies in T cells [38]. T cells expressing a phophataseinactive SHP-2 variant develop normally but generate enhanced immune responses. It has been shown previously that Fc $\gamma$ RIIb co-engagement with the BCR leads to dephosphorylation of CD19 and Gab-2 [39,40]. An increased integration of SHP-2 might be the explanation for this observation. However, the exact role of SHP-2 in Fc $\gamma$ RIIb-mediated negative regulation requires further investigation.

Consistent with enhanced recruitment of the PI3K antagonist SHIP in BCR/FcyRIIb-co-stimulated cells Grb2-mediated integration of the positive PI3K regulators p 85 and Vav-1 is markedly reduced. Hence Fc $\gamma$ RIIbco-engagement appears to shift the whole PI3K regulation from activation to inhibition. Notably, Grb2 interaction with these regulators is $\mathrm{SH} 3$ domain-dependent as for SHP-2 and SHIP-1 [19,41-44]. This divergent signal integration of constitutively interacting proteins is probably based on the fact that stable multi protein complex formation often requires bidentated interactions. The Fc $\gamma$ RIIb-dependent SHIP recruitment discussed above is a very obvious example for this mechanism. It appears possible that integration of positive PI3K regulators is stabilized by Grb2 in context with the co-receptor CD19 in a similar way. CD19 is known bind the SH2 domains of Grb2 and p85 [18,45] and together with Vav and BCAP it orchestrates full PI3K activation [46]. CD19 tyrosine phosphorylation has been reported to be diminished after BCR/Fc $\gamma R$ IIlb co-ligation [39]. Consistently, we found decreased binding of Grb2 to CD19 leading to reduced subsequent recruitment of PI3K activators and a reduction of these proteins within the Grb2 interactome. Hence, FcyRIIb coengagement appears to reduce the amount of PIP3 at the cytosolic side of the plasma membrane by both diminished PI3K activity and enhanced integration of SHIP (for summary, see Fig. 5).

Beyond marked alterations of PI3K regulation our analysis revealed Fc $\gamma$ RIIb-dependently decreased Grb2 binding to PTPRA, Snx-18, ARHGAP-12 and ASAP1. These proteins have not been studied in B cells so far and their putative function can only be deduced from studies in other cell types. Snx-18 and ASAP1 have been shown to regulate intracellular vesicle trafficking [47-49]. PTPRA has been shown to regulate focal adhesion kinase (FAK) [50] whereas ARHGAP-12 is a putative modulator of Rho family GTPases [51]. Hence, it is tempting to speculate that these proteins are involved in regulation of BCR-mediated uptake of antigens as reorganization of the cytoskeleton and trafficking is important for this process [52]. Decreased Grb2-mediated integration of these proteins after Fc $\gamma$ RIIb co-engagement would be conclusive as antigen uptake is not required in this situation, but certainly this is a speculative interpretation.

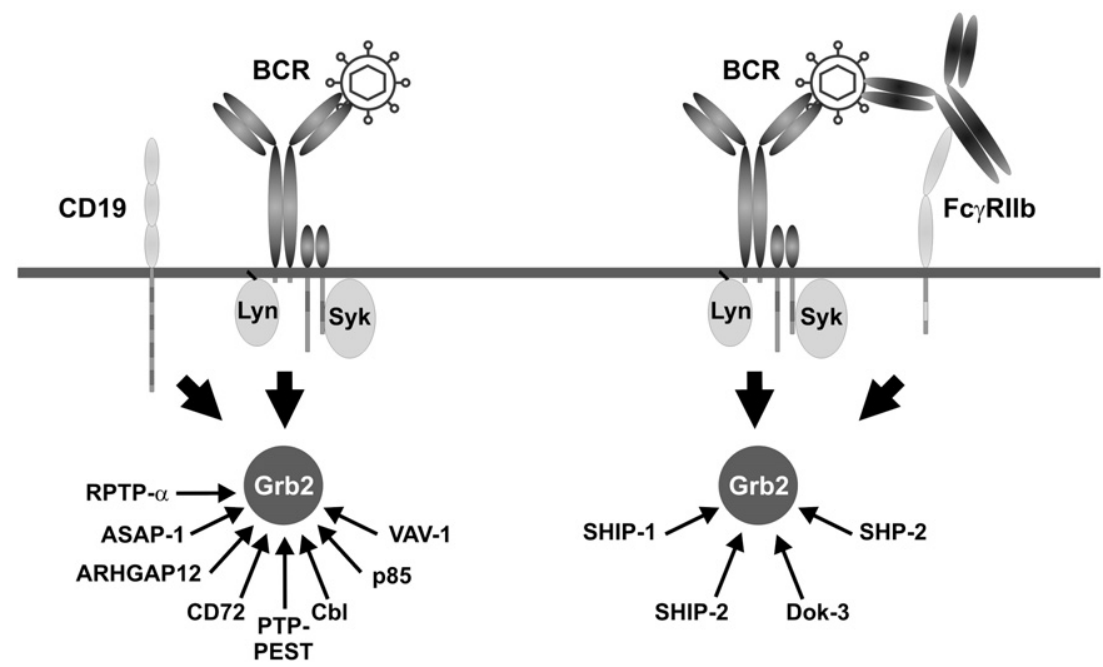

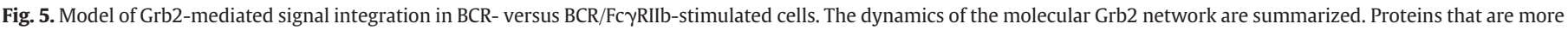

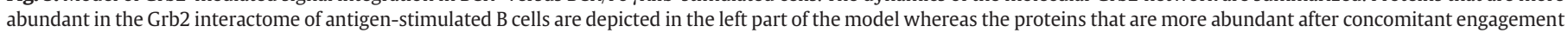
of BCR and Fc $\gamma$ RIIb are shown on the right part of the model. 
Collectively, our approach elucidates the dynamic characteristic of the molecular Grb2 network and emphasizes the complex impact of FcyRIIb co-engagement by shifting signal integration towards negative regulators. Based on these data we propose that differential plasma membrane relocalization of Grb2 by Dok-3 or BCR co-receptors like CD19 is a crucial process to change integration of signal regulators. In conjunction with these transmembrane or plasma membrane-associated proteins Grb2 appears to act as molecular glue to stabilize protein complexes. Here, binding of a Grb2 SH3 domain to a proline rich motif of a signal effector, which itself is bound to the same protein like the Grb2 SH2 domain increases the avidity of the whole protein complex. This could be the mechanism how functionally divergent co-receptors like Fc $\gamma$ RIIb and CD19 utilize Grb2 to integrate either negative or positive regulators of $\mathrm{BCR}$ signaling with the ultimate aim to change the cellular response to BCR engagement.

\section{Acknowledgements}

We thank Dr. André Veillette, Dr. Tony de Franco, Dr. Johannes Lutz, and Dr. Kai Dittmann for sharing reagents and for critical reading of this manuscript, respectively. Johanne Lehne and Uwe Plessmann provided excellent technical help in MS analysis. This work was funded by the DFG research group FOR 521.

\section{Appendix A. Supplementary data}

Supplementary data to this article can be found online at doi:10.1016/j. cellsig.2011.01.015.

\section{References}

[1] M. Engelke, N. Engels, K. Dittmann, B. Stork, J. Wienands, Immunol. Rev. 218 (2007) 235-246.

[2] J. Wienands, J. Schweikert, B. Wollscheid, H. Jumaa, P.J. Nielsen, M. Reth, J. Exp. Med. 188 (4) (1998) 791-795.

[3] C. Fu, C.W. Turck, T. Kurosaki, A.C. Chan, Immunity 9 (1) (1998) 93-103.

[4] C.W. Chiu, M. Dalton, M. Ishiai, T. Kurosaki, A.C. Chan, EMBO J. 21 (23) (2002) 6461-6472.

[5] N. Engels, M. Engelke, J. Wienands, Adv. Immunol. 97 (2008) 251-281.

[6] H. Niiro, E.A. Clark, Nat. Rev. Immunol. 2 (12) (2002) 945-956.

[7] X. Li, R.H. Carter, Eur. J. Immunol. 30 (6) (2000) 1576-1586.

[8] A.C. Lankester, P.M. Rood, G.M. van Schijndel, B. Hooibrink, A.J. Verhoeven, R.A van Lier, J. Biol. Chem. 271 (37) (1996) 22326-22330.

[9] D.A. Fruman, A.B. Satterthwaite, O.N. Witte, Immunity 13 (1) (2000) 1-3.

[10] J.A. Deane, D.A. Fruman, Annu. Rev. Immunol. 22 (2004) 563-598.

[11] J.C. Unkeless, J. Jin, Curr. Opin. Immunol. 9 (3) (1997) 338-343.

[12] L. Nitschke, Curr. Opin. Immunol. 17 (3) (2005) 290-297.

[13] B. Heyman, Annu. Rev. Immunol. 18 (2000) 709-737.

[14] M. Ono, H. Okada, S. Bolland, S. Yanagi, T. Kurosaki, J.V. Ravetch, Cell 90 (2) (1997) 293-301.

[15] M. Ono, S. Bolland, P. Tempst, J.V. Ravetch, Nature 383 (6597) (1996) 263-266.
[16] T. Takai, M. Ono, M. Hikida, H. Ohmori, J.V. Ravetch, Nature 379 (6563) (1996) 346-349.

[17] T. Takai, Nat. Rev. Immunol. 2 (8) (2002) 580-592.

[18] S.R. Brooks, X. Li, E.J. Volanakis, R.H. Carter, J. Immunol. 164 (6) (2000) 3123-3131.

[19] I. Isnardi, R. Lesourne, P. Bruhns, W.H. Fridman, J.C. Cambier, M. Daeron, J. Biol. Chem. 279 (50) (2004) 51931-51938.

[20] K.L. Otipoby, K.E. Draves, E.A. Clark, J. Biol. Chem. 276 (47) (2001) 44315-44322.

[21] B. Stork, K. Neumann, I. Goldbeck, S. Alers, T. Kahne, M. Naumann, M. Engelke, J. Wienands, EMBO J. 26 (4) (2007) 1140-1149.

[22] C.H. Ng, S. Xu, K.P. Lam, Blood 110 (1) (2007) 259-266.

[23] B. Stork, M. Engelke, J. Frey, V. Horejsi, A. Hamm-Baarke, B. Schraven, T. Kurosaki, J. Wienands, Immunity 21 (5) (2004) 681-691.

[24] N. Engels, L.M. Konig, C. Heemann, J. Lutz, T. Tsubata, S. Griep, V. Schrader, J. Wienands, Nat. Immunol. 10 (9) (2009) 1018-1025.

[25] K. Neumann, T. Oellerich, H. Urlaub, J. Wienands, Immunol. Rev. 232 (1) (2009) 135-149.

[26] A. Grabbe, J. Wienands, Blood 108 (12) (2006) 3761-3768.

[27] W.M. Kavanaugh, D.A. Pot, S.M. Chin, M. DeuterReinhard, A.B. Jefferson, F.A. Norris, F.R Masiarz, L.S. Cousens, P.W. Majerus, L.T. Williams, Curr. Biol. 6 (4) (1996) 438-445.

[28] S. Lemay, D. Davidson, S. Latour, A. Veillette, Mol. Cell. Biol. 20 (8) (2000) 2743-2754

[29] T.M. Saxton, I. van Oostveen, D. Bowtell, R. Aebersold, M.R. Gold, J. Immunol. 153 (2) (1994) 623-636

[30] S.L. Harmer, A.L. DeFranco, J. Biol. Chem. 274 (17) (1999) 12183-12191.

[31] S. Sarkar, K. Schlottmann, D. Cooney, K.M. Coggeshall, J. Biol. Chem. 271 (33) (1996) 20182-20186.

[32] V.L. Ott, I. Tamir, M. Niki, P.P. Pandolfi, J.C. Cambier, J. Immunol. 168 (9) (2002) 4430-4439.

[33] J.D. Robson, D. Davidson, A. Veillette, Mol. Cell. Biol. 24 (6) (2004) 2332-2343.

[34] E. Muraille, P. Bruhns, X. Pesesse, M. Daeron, C. Erneux, Immunol. Lett. 72 (1) (2000) 7-15.

[35] Z.Q. Shi, D.H. Yu, M. Park, M. Marshall, G.S. Feng, Mol. Cell. Biol. 20 (5) (2000) 1526-1536.

[36] W. Li, R. Nishimura, A. Kashishian, A.G. Batzer, W.J. Kim, J.A. Cooper, J. Schlessinger Mol. Cell. Biol. 14 (1) (1994) 509-517.

[37] R.J. Salmond, D.R. Alexander, Trends Immunol. 27 (3) (2006) 154-160.

[38] R.J. Salmond, G. Huyer, A. Kotsoni, L. Clements, D.R. Alexander, J. Immunol. 175 (10) (2005) 6498-6508.

[39] K.L. Hippen, A.M. Buhl, D. D'Ambrosio, K. Nakamura, C. Persin, J.C. Cambier, Immunity 7 (1) (1997) 49-58.

[40] G. Koncz, G.K. Toth, G. Bokonyi, G. Keri, I. Pecht, D. Medgyesi, J. Gergely, G. Sarmay, Eur. J. Biochem. 268 (14) (2001) 3898-3906.

[41] M.D. Ware, P. Rosten, J.E. Damen, L. Liu, R.K. Humphries, G. Krystal, Blood 88 (8) (1996) 2833-2840.

[42] L. Wong, G.R. Johnson, J. Biol. Chem. 271 (35) (1996) 20981-20984.

[43] M. Nishida, K. Nagata, Y. Hachimori, M. Horiuchi, K. Ogura, V. Mandiyan, J. Schlessinger, F. Inagaki, EMBO J. 20 (12) (2001) 2995-3007.

[44] Z.S. Ye, D. Baltimore, Proc. Natl Acad. Sci. USA 91 (26) (1994) 12629-12633.

[45] S.R. Brooks, P.M. Kirkham, L. Freeberg, R.H. Carter, J. Immunol. 172 (12) (2004) 7556-7564.

[46] Y. Aiba, M. Kameyama, T. Yamazaki, T.F. Tedder, T. Kurosaki, Blood 111 (3) (2008) 1497-1503.

[47] Park J, Kim Y, Lee S, Park JJ, Park ZY, Sun W, Kim H, Chang S. J. Cell Sci. 123 (Pt 10) (2010) 1742-1750.

[48] K. Haberg, R. Lundmark, S.R. Carlsson, J. Cell Sci. 121 (Pt 9) (2008) 1495-1505.

[49] P.A. Randazzo, J. Andrade, K. Miura, M.T. Brown, Y.Q. Long, S. Stauffer, P. Roller, J.A. Cooper, Proc. Natl Acad. Sci. USA 97 (8) (2000) 4011-4016.

[50] J. Su, M. Muranjan, J. Sap, Curr. Biol. 9 (10) (1999) 505-511.

[51] Z. Zhang, C. Wu, S. Wang, W. Huang, Z. Zhou, K. Ying, Y. Xie, Y. Mao, Int. J. Biochem. Cell Biol. 34 (4) (2002) 325-331.

[52] F.D. Batista, N.E. Harwood, Nat. Rev. Immunol. 9 (1) (2009) 15-27. 